\title{
Towards Short-Term Content Adaptation
}

\author{
Michela Acquaviva Marco Benini \\ Dipartimento di Informatica e Comunicazione \\ Università degli Studi dell'Insubria \\ via Mazzini 5 , \\ IT-21100 Varese, Italy \\ \{michela.acquaviva, marco.benini\}@uninsubria.it
}

\begin{abstract}
Recent works in the E-Learning field tend to focus on the learner, leaving the representation and treatment of contents in the background. One of the principal actors that contribute on this focus shift is the adoption of adaptive techniques that profile learners and adjust the contents according to the inferred profiles. In contrast with most adaptive approaches, this work introduces a short-term adaptive strategy whose aim is to capture the instantaneous interests of users. The proposed model fills the temporal gap that other adaptive strategies leave open. In fact, as far as we know, all proposed adaptive strategies have been conceived to deduce complex and accurate profiles in a long amount of time. Instead, the proposed strategy operates observing few actions to deduce a rough profile that is useful to provide continuos adaptive behaviour even if a more precise profile has not yet being constructed.
\end{abstract}

Keywords: Short-term adaptive behaviour, User profiling, Information filtering

\section{INTRODUCTION}

Shortly after the born of the World Wide Web, the potential of Internet-based, computer-supported learning has become unveiled. The ability of users to access an enormous, widespread amount of information suggested the possibility to provide online courses to whoever needs instruction. Universities and, more in general, teaching institutions began to provide organised online courses and teaching or tutoring services.

This wealth of instructional opportunities caused the development of specific platforms whose aim was to provide the instruments to build and to consult learning services. The whole of these instruments and their underlying techniques gave raise to a new area of study and research known as E-Learning.

The distinctive character of E-Learning is the physical distance between students and teachers. In fact, E-Learning represents a peculiar system of training that, thanks to different tools and special formal procedures (including synchronous and asynchronous instruments as chat, forum, e-mail...), integrates the traditional classroom education: it breaks the bond of time and space providing that who transfers the knowledge and who receives it should be, simultaneously, in the same place. On the contrary, in the E-Learning approach, the teaching contents are available and usable in moments and places chosen by the users.

Besides, the task the new communication technologies are entrusted with is to reduce the distances and to give a sense of proximity and community of interest, in order to allow an effective interaction between learners and teachers.

Hence, a great effort has been spent to improve the quality of course material, by adding graphics, animations and speech. For this reason, multimedia achieved a growing importance in the E-Learning technologies, in order to make courses more appealing, clearer and simpler to understand, paying attention to guarantee usability [12] and accessibility [10] to the educational contents. 
This new complex and very expensive approach (either in terms of effort and time, or in terms of money), induced content designers and developers to make teaching material modular and reusable on many platforms. In particular, modularity led to Learning Objects (LOs) [3], small units of teaching information, represented according to widely accepted standards, such as SCORM [2], LOM [20], etc.

Nowadays, standards are well established in the multimedia field and in the E-Learning community and most platforms support them, making possible to reuse educational material.

Despite this evolution of contents, there is a general dissatisfaction with the benefits of teaching and learning on the Internet. Statistical analyses, e.g., [4], tell that E-Learning is not effective since most students do not reach an acceptable level of understanding.

On the other side, teachers have great difficulties in constructing online courses and assembling good teaching material because they perceive LOs as intrinsically independent from their context, even if specifically developed for this purpose. Moreover, in comparison with the traditional lessons held in a classroom, in an E-Learning environment students are not necessarily involved in an educational process tailored to their needs. For example, a programmer wishing to learn about a new technology has a different goal than a marketing consultant that needs an overview of the same technology.

In a traditional educational experience, when a teacher selects the learning material and exposes a course, he naturally perceives the level of the classroom and tends to adapt his pace and his style to what is best suited to students' abilities and preferences, while in the E-Learning context the material is not individualised. E-Learning products are not able, until now, to adapt the content to these different needs, overcoming this lack of personalisation.

Henceforth, an adaptive behaviour in the E-Learning process seems to be the way to move from a content-centred conception of the learning support, to a learner-centric vision, where the material is instrumental to aid the understanding. The "adaptive behaviour" is, in general, the ability to modify the content according to a model of the learner.

With respect to modifications on the content, we distinguish among:

1. changes in the presentation: it means to modify the graphical appearance of LOs, or to render text in speech (mostly useful to solve accessibility problems and to address usability problems as well, see [12]);

2. changes in the real content: it implies modifications in the semantics of LOs, thus they have a deeper impact on the LOs design. Of course, the learning material should be designed for this purpose, adding another level of difficulty in the development of LOs;

3. changes in the content description: it means to act on the metadata [21] used to classify LOs. Since metadata are represented according to standards [19], an application can uniformly manipulate them in order to move a LO in the classification the learner consults to retrieve material of interest.

To adapt LOs an application needs a model of the learner that evolves during time: in this way the learner actions are interpreted to update the model and to propose the best suited material to his inferred needs.

Hence, a very structured, and therefore complex model of the user permits a precise action on LOs, by manipulating both the presentation and the metatdata of LOs, and appears to be quite effective, as reported in the numerous experiences discussed in Section 3.

Despite this fact, due to the youth of the research on adaptive applications, there is not yet a reference model that allows standardisation and, thus, the possibility to reuse adaptive components in different frameworks. Hence, a great effort has been spent in exploring different models to formalise many aspects of the user interests and capabilities, and to plan suitable adaptations on LOs. 
There is a concentration of research and experimentation on long-term profiling, that is, on the instantiation of a learner model over a long time, accumulating indications of his interests and capabilities over a range of weeks or months.

Although these approaches produce interesting results, see again Section 3, they are also slow, and thus discouraging for learners that expect a rapid understanding of their needs by the ELearning system.

On the contrary, the purpose of this work is to present a model for a short-term adaptive behaviour, filling the gap that seems to lie between the user expectations and the technological offer.

The proposed model is part of a wider E-Learning project, VICE [1], that we will introduce in Section 2. A comparison among the VICE project and other adaptive approaches in the ELearning area can be found in Section 3. The core of the mathematical formalisation of the shortterm adaptive model is presented in Section 4. Since VICE is an ongoing project, experimental results are not available in a form that allows a deep discussion, so they are briefly presented in the Conclusions, along with future directions.

\section{THE VICE PROJECT}

The VICE (Virtual Continuing Education) project [1] aims to develop an innovative methodology and platform to build high-level E-Learning applications with a strong technological support.

VICE wants to support instruction in the ICT (Information and Communication Technologies) area, and in particular, the project focuses on assisting the development and growth of small business firms.

The small business firms who are partners in the VICE project are in the Italian geographical region known as Salento, and have been chosen since they form an homogeneous base where to test the validity of the VICE products. Their role is to experiment on the field the VICE platform on a series of LOs developed to instruct professionals about the ICT themes and about the security problems. The teaching method will follow the blended approach, i.e., aside a small traditional teaching activity, most of the instructional process takes place online.

\subsection{Goals}

The VICE project is strongly based on the Semantic Web technology [7] and it uses Artificial Intelligence techniques to cope with the adaptive aspects of LOs fruition. Hence, the VICE platform provides a repository for LOs properly enriched with suitable metadata along with a formal semantics that gives meaning to metadata.

Therefore, the content of the repository can be used not only by human users, but also by artificial agents. These agents support semantic navigation of the repository content, automatically construct lessons, and verify that a lesson is composed of LOs satisfying some pedagogical criterion.

In this wide perspective, the VICE project wants to develop an innovative platform that allows the creation, storage and access to LOs and their metadata, with a special attention to reusability of learning components. Moreover, the VICE platform wants to provide support to the creation of personalised courses and lessons, automatically adapted to the cognitive needs of learners.

The MILOS (MultImedia Learning Object Server) component provides the necessary storage and querying mechanisms. MILOS is based on a powerful multimedia database, specifically developed to represent and to manipulate LOs represented in the XML technologies and following the SCORM standard [2]. 
The choice of $\mathrm{XML}$ as the base representation language allows the integration of E-Learning standards, like SCORM, with multimedia standards, like MPEG-7 [22] and SMIL [5], but also with Semantic Web standards, like RDF [21] and OWL [26].

Moreover, since the repository is $X M L$ based, it benefits of the $X M L$ representation technologies, as XSchema [14], and of the XML querying languages, XPath [11] and XQuery [9].

The repository provides the following access methods to end-users:

1. querying the content: the user may search LOs matching some criterion on metadata, or even on the data content, e.g., text in slide notes;

2. navigation: since LOs are semantically related by means of their metadata, e.g., prerequisites or level of difficulty, the user can browse the repository following these semantic links;

3. semantic or pedagogical querying: the user may ask for courses, i.e., sequences of LOs, satisfying some didactic goal that covers not only the general topic, but also the preferred pedagogical style.

Moreover, the access to the repository is adaptive, that is, whenever the user poses a query or follows a link, the system analyses this request to individuate a profile. Then the system transforms the retrieved answer according to the inferred interests and preferences.

Another goal of the VICE project is the ability to federate with analogous systems. In fact, there is a live discussion in the research community on the possibility to construct open networks of learning services, that share their repositories and, to some extent, their tools. For example, the Canadian project presented in [19] deals with the problem of integrating LOs in heterogeneous formats, by providing flexible searching capabilities.

In this context, VICE plans to be a candidate architecture for nodes in future federated networks, since it is already designed to support the proposed models and methods to facilitate interoperation.

\subsection{The Pedagogical Wizard}

The Pedagogical Wizard is the component in the VICE project that realises the semantic or pedagogical querying activity, see Figure 1.

It forces the LOs representation to support pedagogical and semantic metadata that are interpreted according to the wizard knowledge, organised as a coordinated set of ontologies written in the OWL language [26].

The PEDAGOGICAL WIZARD interprets LOs as the necessary information to support a didactic process. Hence, by means of a pedagogical taxonomy, it can classify LOs according to their style, to their level of difficulty and to their relative importance in a specific learning process.

Therefore, the PEDAGOGICAL WIZARD benefits of this information to compose LOs in lessons and courses, with the warranty that the composition is uniform and balanced with respect to the pedagogical criteria specified by the user. An intelligent agent that acts by planning the lesson or course with Artificial Intelligence techniques performs this composition activity.

A side effect of the knowledge available in the ontologies involved in the planning activity is that the repository can be inspected, i.e., queried or browsed, following the semantic categories (the ontological relations) that describe the knowledge. Thus, the ontological representation provides the support for the semantic and pedagogical querying feature.

In addition, the knowledge developed to support planning helps the generation of the adaptive behaviour that is the focus of this paper. 


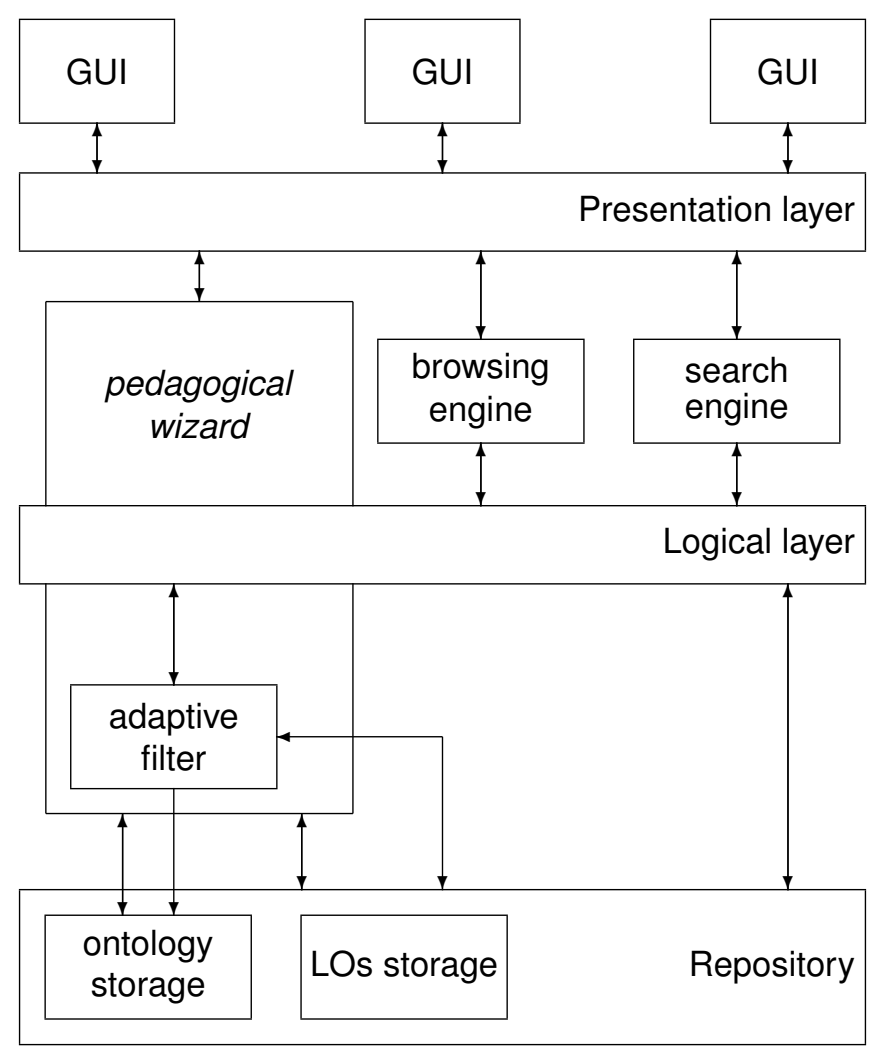

FIGURE 1: Architecture of the VICE platform.

The represented knowledge, although coded in the form of OWL ontologies, wants to embody the common knowledge an expert may have of the E-Learning process in general, and of the specific instructional domains the LOs in the repository cover. It is evident that this sort of knowledge is mostly qualitative, sometimes incomplete, and often imprecise or approximated.

Therefore, to deal with incomplete, qualitative and imprecise pieces of information, the ontologies have been developed in a reasoning framework that is intrinsically fuzzy, see [18]. To support the adaptive behaviour, which is based on a topological model, see Section 4, a special attention has been given to the compatibility of the fuzzy and topological approaches, obtaining a fuzzy logical system [25] that can be mapped into a reasonable topological space.

A subtler goal of the PEDAGOGICAL WIZARD stems from the fact that currently available Semantic Web applications are still prototypes. Hence, understanding how to structure and to manipulate the knowledge in a wide application like the wizard is something worth studying in itself. The results of this activity will be a series of guidelines that explain how to codify the knowledge involved in the learning processes, aiming to help the extension of the wizard to other domains of application.

\subsection{The adaptive behaviour}

In order to construct automatic agents that search and compose LOs in an effective and coherent way, it is necessary to have an integrated ontological system that refers to all elements constituting the application domain.

Specifically, the needed ontologies cover (i) the contents - to correctly extract and navigate the objects in the repository; (ii) the users - to personalise the instructional process; (iii) the learning goals; (iv) the taxonomies of the LOs and (v) the pedagogical criteria codifying the teaching styles. 
The adaptive agent uses these ontologies to derive the set of attributes it operates on, and to represent queries as elements in its manipulation space. Precisely, the adaptive agent is a filter that intercepts queries to the repository and uses them to construct a user profile. Then, it transforms the answer of the repository according to the adaptive strategy. The profile is a model of the querying user, which is devoted to code his interests and characters. While interests are intended to be short-term goals the user wants to fulfil, characters are considered as long-term information describing the user preferences and capabilities.

In this paper, we will focus on the short-term behaviour that constitutes the real novelty in our adaptive approach. In fact, the long-term adaptive model is a natural extension of the short-term model, and is comparable with many existing approaches, see Section 3.

The goal the adaptive filter wants to meet is the convergence of the transformation process. Precisely, in the hypothesis that a sequence of queries represents an attempt to satisfy an interest of the user, we expect that every query in the sequence refines the interest, until a satisfying result is found. Hence, the transformation process should result in a series of answers that are closer and closer to the satisfaction of the user interest, and this series of answers should converge with the same speed as the sequence of queries.

Therefore, the filter must be able to segment the flow of queries from a user in sequences each of one converging to the full expression of a single interest, until the interest gets satisfied. In other words, the filter should sense the changes of interest in the flow of queries issued by a user.

The filter provides families of transformations, such as ordering the elements of a multiple answer according to their closeness to the user interest. These transformations are parameterised with the user profile.

The property the filter is called to preserve is informally stated as "to a small variation in the profile, a small variation in applied transformation corresponds", hence the need for a mathematical model. In fact, the model is more precise and what it does really preserve is the convergence speed, see Section 4 for details.

The cognitive aspects have a fundamental role in the success of the adaptive process. In fact, the categories used to classify user interests and characters have a fundamental role in the definition of the granularity of the profile. Since every interest is represented as a weighted set of categories, the filter can recognise only interests that fit into this representation. Hence, a large number of well-coordinated categories that uniformly covers the whole application domain greatly enhance the filter understanding of the user interest.

\section{RELATED WORKS}

Since the interests of users in different approaches to the educational resources is a fundamental subject matter of adaptivity, several applications have paid much attention to define a set of instructional strategies in order to meet users' individual learning styles and preferences and, thus, to improve their performances.

Therefore, the mainstay of such applications is to provide students with different presentation of learning resources, as in TANGOW [8], THEO [16], ARTHUR [17], or within an experimental virtual campus at the Open University of Catalunya [23], according to parameters strictly related to their profile, including the actions they perform while attending their tasks, and their individual preferences about learning strategies.

Another interesting solution is based on the complementary pair of authoring systems AHA!, described as the adaptive hypermedia engine pull, and MOT, described as the adaptive hypermedia authors push [28], whose main goal is to provide authors with specific tools that help them to supplement their adaptive applications by designing as many assortments and combination of learning styles as possible; individual learning styles are assessed through a registration form where users select their favourite instructional strategy. 
What is most noteworthy in this project, the users' browsing behaviour allows the system to improve its performance by inferring their preferences and keeping them up to date from time to time.

Besides performing an effective learning experience based on individualised instructional strategies, one of the main problems students and teachers have to deal with is how to select the most appropriate topics, or learning resources, and arrange them in a coherent, focused path.

The cooperation between IBM and MIT gave rise to a pilot study, whose core is the DYNAMIC ASSEMBLY ENGINE [15], to automatically assemble LOs in personalised paths, by ordering the resources in a logical sequence. Actually, the process flow provides that, once a query has been submitted, the system returns a list of the most relevant results matching the users' requirements, according to their educational role.

The main concern of this work is to find a solution not only to select the most appropriate resources to a specific learning path, but to connect them in an well-organised corpus too, tailored to specific cognitive needs.

The framework of the DYNAMIC ASSEMBLY ENGINE is particularly close to VICE, see Section 2, since a XML federated repository, where learning resources are stored, and a strict correspondence of LOs content and their metadata to users' needs work together to make this approach feasible.

The accuracy of the system is guaranteed by the knowledge the application has of users' queries, ad the coherence of the path is achieved by presenting objects that are closely related with one another, meeting the search parameters.

The German prototype ELENA [13] is another valuable research work in the domain of personalised supports for students in an E-Learning network, in order to create an adaptive Semantic Web environment, and is based on the EDUTELLA P2P infrastructure [24].

The main contribution of ELENA is the creation of smart spaces for learning, defined as educational service mediators, based on Semantic Web and Artificial Intelligence technologies. These spaces provide students with an exhaustive description of those learning resources that meet their profile, by allowing them to access any kind of repository which is connected on the network.

Therefore, the German team, committed in creating an open network of repositories of LOs in the sense of [19], aims at setting up an organised network of learning and knowledge management systems, so that students can access resources, lessons and courses according to their individual profile.

The keystone of this project, the PERSONAL LEARNING ASSISTANT, is expected to present a focused list of learning services. The action performed by the ASSISTANT refers to the user dynamic profile in order to extend queries by adding restrictions, variables, preferences, goals, etc., and to customise search results.

As this section has shown, there are several works on adaptivity. The close likeness between all these works and VICE is due to the fact that the adaptivity the filter provides in our work concerns the capability to take in account all the users' features in order to customise educational contents and make them fulfil their cognitive needs.

In our opinion, in comparison with the other projects, the real noteworthy aspect of VICE is that the filter is conceived to address the short-term behaviour of users, while the adaptive approaches described so far have been developed to exhibit a long-term understanding. As far as we know, no short-term approach to adaptivity appears in the E-Learning literature. 


\section{A SHORT-TERM ADAPTIVE MODEL}

The adaptive filter is based upon two algorithms: the analyser that extracts information from the query and updates the user profile, and the transformer that chooses a transformation, parameterised by the user profile, to apply to the result. The filter receives a query from the user; then, it analyses the query with the analyser algorithm, and it forwards the query to the repository; finally, it modifies the answer from the repository by means of the transformer algorithm.

The goal of the adaptive strategy performed by the filter is to exhibit two behaviours: convergence and changes of interest. In other words, the series of transformed results the filter produces when the user supplies a convergent sequence of queries must converge. Hence, if the sequence of queries approximates the user interest, then the series of results must be closer and closer to the satisfaction of that interest.

On the other hand, the notion of change of interest models the situation when the user stops a sequence of queries and starts a new one, which focuses on a different interest.

\subsection{Modelling user profiles}

The user is modelled as a point in the profile space, denoted as $U_{\text {profile, }}$, the cube with edge $[-1,1]$ in the $n$-th dimensional Euclidean space.

Every coordinate of this space is uniquely associated with an attribute. Thus, the user profile, a point in the profile space, is a set of pairs of the form (attribute, $w$ ), where $w$ is the weight of the attribute, denoting how strong is the user interest in the attribute.

For example, the pair (Java, 0.7) indicates a strong interest in the Java subject, while (math, -0.2$)$ denotes a weak lack of interest in the topics related to mathematics.

Being a subspace of the Euclidean space, the profile space is equipped with a norm that measures the length of a profile $\left(p_{1}, \ldots, p_{n}\right)$ :

$$
\left\|\left(p_{1}, \ldots, p_{n}\right)\right\|=\sqrt{\sum_{i=1}^{n} p_{i}^{2}}
$$

Obviously, the associated distance function is $d(a, b)=\|a-b\|$ as usual, see,e.g., [27].

Moreover, the zero vector represents the neutral profile, modelling the user deprived from his interests.

The important point in our model is that a query can be interpreted as a profile.

In fact, there is a direct correspondence between attributes and classes in the ontologies of the Pedagogical Wizard. Thus, since an ontology is also a semantic network [26], one can mark the classes corresponding to the queried metadata.

Precisely, the marking process assigns a fixed weight to a class, say 1 if the query requires satisfying LOs to be members of the class, and -1 if it does not. All other classes are marked with 0 .

We assume that the relations among classes have been weighted by the human expert who developed the ontology. Therefore, the whole network, initially marked as explained, tries to propagate markings to every class, eventually adjusting calculated weights, see [6] for details, until it becomes stably weighted. Hence, the query profile is composed by the attributes weighted as their corresponding classes.

Therefore, given a sequence of queries $q^{1}, \ldots, q^{n}$, it is possible to associate to every query a corresponding profile, obtaining a sequence of query profiles $q p^{1}, \ldots, q p^{n}$. Thus, given an 
initial user profile $p^{0}$, the sequence of queries $q^{1}, \ldots, q^{n}$ generates the sequence of user profiles $p^{0}, \ldots, p^{n}$ with the rule that

$$
p^{i+1}=\alpha p^{i}+\beta q^{i},
$$

where $\alpha+\beta=1$ and $\alpha, \beta>0$.

The choice of calculating a new user profile by means of a linear combination of the old user profile with the query profile is simply justified by the fact that the constraints on $\alpha$ and $\beta$ impose that the linear combination is a weighted mean of the two vectors.

Consequently, the rule that identifies a change of interest is a lack of convergence in the sequence. A way to locally measure the convergence of the generated profile sequence, is to observe if $\left\|p_{i+1}-p_{i}\right\|>\delta / f(i)$, where $\delta$ is a constant threshold value, and $f$ is a monotone increasing function. For example, if $f(i)=i$ then a sequence that does not violate the constraint is converging with linear speed; while, if $f(i)=\log i$, the sequence converges with logarithmic speed. In general, $f$ measures the required convergence speed of generated profiles.

The analyser algorithm converts the input query $q^{i}$ to a profile $q p^{i}$, and then it calculates the new profile $n$ as the weighted average of $q p^{i}$ and $p^{i}$, the current profile. If the distance between $n$ and $p^{i}$ is too big, i.e., greater than $\delta / f(i)$, then the analyser restarts a new query sequence, otherwise $p^{i+1}$ becomes equal to $n$.

\subsection{Modelling transformations}

The transformer algorithm is designed according to the principle that a small variation in the user profile induces a small variation in the applied transformation.

Thus, we assume that the filter is equipped with a set of transformations, and one of them is applied to the result according to the query and the user profile, as previously explained.

The transformation applied to the result depends on the query structure. To capture this dependency the set $Q$ of possible queries is partitioned by an equivalence relation $\rho$. Hence, the transformation space is the disjoint union of transformations:

$$
\Theta=\bigsqcup_{x \in Q / \rho}\left\{\theta_{x}(p) \mid p \in U_{\text {profile }}\right\} .
$$

The functions $\theta_{x}: U_{\text {profile }} \rightarrow\left(U_{\text {answer }} \rightarrow U_{\text {answer }}\right)$ are the transformations. They act by generating a concrete function from a user profile, that, when applied to a result, produces the transformed result. The result is considered a point in the space $U_{\text {answer }}$, the set of possible answers, usually identified with the set of valid XML documents.

The link between the transformation space and the profile space is given by a constraint that ensures the preservation of convergence. Let $x$ in $Q / \rho$ be the selector of a transformation, let $a$ in $U_{\text {answer }}$ be an answer from the repository, then, we impose that, for every $p a$ and $p b$ in $U_{\text {profile, }}$,

$$
d_{x}\left(\theta_{x}(p a)(a), \theta_{x}(p b)(a)\right) \leq K d(p a, p b),
$$

where $d$ is the distance function in the profile space, $d_{x}$ is the distance function associated with the $\theta_{x}$ transformation in the answer space, and $K$ is a (small) constant, possibly depending on $a$ and $x$.

Henceforth, a transformation $\theta_{x}$ is admissible iff it is possible to associate a distance function $d_{x}$ that satisfies the constraint 1 . In fact, if the distance function $d_{x}$ is chosen such that the metric space $U_{\text {answer }}$ is a Banach space, where every Cauchy sequence is convergent [27], then the constraint 1 is a direct formalisation of the sentence "a small variation in the profile space induces a small variation in the transformation", since a small variation in the distance between two profiles is reflected in a small variation between the results as calculated by the same transformation applied to the same answer from the repository. In other words, the constraint ensures that there 
is a homeomorphism between the profile space and a suitable subspace of the transformation space.

As an example of admissible transformation, we consider the transformation that order a list of answers according to their proximity with the user interests.

Precisely, the ordering transformation operates by sorting the list of answers to the query according to the user profile. To every element $r_{i}$ of the list $\left(r_{1}, \ldots, r_{n}\right)$ is associated a point $p r_{i}$ of $p r=\left(p r_{1}, \ldots, p r_{n}\right)$ in the profile space, in a similar way as already described for converting queries into profiles. The sorting key may be any continuous function $f: U_{\text {profile }} \times U_{\text {profile }} \rightarrow \mathcal{R}$, where $\mathcal{R}$ is the set of real numbers. Since the function $f$ is applied to the pair $\left(p r_{i}, p\right), p$ being the user profile, the list is sorted according to $f$ and $p$, as required.

Moreover, the ordering transformation satisfies the constraint 1, since, posing $d_{x}(a, b)$ as the minimal number of element swappings needed to transform the list $a$ into the list $b$, it follows that $d_{x}$ is, indeed, a distance function. Since $f$ is continuous, but sorting is a discrete function, a sufficiently small value of $d(p a, p b)$ implies that $\theta_{x}(p a)(a)=\theta_{x}(p b)(a)$, thus, calling $\epsilon$ such value, it follows that $d_{x}\left(\theta_{x}(p a)(a), \theta_{x}(p b)(a)\right) \leq n / \epsilon$, because no more than $n$ swaps are needed to transform a list of length $n$ into any of its permutations. Thus, posing $K=n / \epsilon$, the constraint 1 immediately follows.

\section{CONCLUSIONS}

The mathematical model presented in Section 4 provides a simple framework for the short-term adaptation process. Due to lack of space, we avoided to show the mathematical analysis of the model, but it is possible to prove that the filter is correct: if a series of queries converges, then the corresponding generated answers are transformed in a way that approximates better and better the user interest. Moreover, the rule to model the change of interest guarantees that the converging process is reasonably fast.

Since the user model is very simple, being just a list of weighted attributes, the adaptive behaviour is rough. Nevertheless, in a short-term context, the speed of adaptation compensates this roughness, thus giving the user the illusion that the system really understands his interests. A quantitative measure of this understanding is the fact that the convergence speed in the transformation space is, essentially, equal to the convergence speed in the profile space. Out of the formal aspect, it means that our adaptive strategy is as reactive as the user. In this sense, we claim that our model operates in the short-term range.

The VICE project is still ongoing and thus the system as a whole is not yet available to experimentation on the field. Hence, the adaptive filter has not been tested in its natural framework, i.e., a complete, fully featured E-Learning application used by professionals wanting to extend and to refine their knowledge.

In the moment being, the adaptive filter underwent the testing phase and has been experimented on small examples of repositories with a limited interface.

The results collected so far are encouraging, since users recognise a better understanding of their needs by the system, but the statistical relevance of our experiments does not permit a significant comparison with related approaches. In fact, our repositories are still too elementary, the number of learners is far too limited and the navigational instruments, that generate the queries the filter uses as observational input, are too poor.

Still, we believe that in the near future, when the VICE project will be completed and experimental results could be collected, the value of a short-term adaptive strategy in a complex E-Learning system will become evident.

Future directions, apart the collection of experimental data, will cover the extension of the mathematical model to other admissible transformation classes. 
Moreover, the presented model relies on the assumption to have a fuzzy ontological system that supports the filter action. We have preliminary results that show how to eliminate the dependency on a fuzziness that appears difficult to realise in general contexts.

Finally, although conceived in the framework of E-Learning, the described adaptive behaviour could be extended to other classes of applications in need of adaptive treatment of information, see, e.g. [29].

This work has been supported by the project "VICE: Comunità virtuali per la formazione", MURSTCNR 449/97.

\section{REFERENCES}

[1] Acquaviva M., Benini M., VICE: E-Learning nell'era del semantic web, in Atti di EXPO ELearning 2004, Ferrara, 2004. In Italian.

[2] Advanced Distributed Learning (ADL) Technical Team, Sharable Content Object Reference Model (SCORM) Version 1.2, 2001.

[3] Anderson T., Elloumi F., Theory and Practice of Online Learning, Athabasca University, 2004.

[4] Anee-Assinform, Osservatorio 2004, 2004. In Italian. http://www.anee.it/ricerche/osservatorio04/default.asp

[5] Ayars J., Bulterman D., Cohen A, et al., Synchronized Multimedia Integration Language (SMIL 2.0), W3C Recommendation, 2001.

[6] Benders E.A., Mathematical Methods in Artificial Intelligence, IEEE Computer Society Press, 1996.

[7] Berners-Lee T., Handler J., Lassila O., The semantic web, Scientific American, 2001.

[8] Carro R., Pulido E., Rodriguez P., Dynamic generation of adaptive internet-based courses, Journal of Network and Computer Applications, 22:249-257, 1999.

[9] Chamberlin D., Fernández M.F., et al., XQuery from the Experts, H. Katz editor, Addison Wesley, 2003.

[10] Chisholm W., Vanderheiden G., Jacobs I., Web Content Accessibility Guidelines 1.0, W3C Recommendation, 1999.

[11] Clark J., DeRose S., XML Path Language (XPath) Version 1.0, W3C Recommendation, 1999.

[12] Dix A., Finley J., Abowd G., Beale R., Human Computer Interaction, 2nd edition, Prentice Hall, 1998.

[13] Dolog P., Sintek M., Personalization in Distributed e-Learning Environments, in [30].

[14] Fallside D., XML Schema part 0: Primer, W3C Recommendation, 2001.

[15] Farrell R., Libburd S.D., Thomas J.C., Dynamic Assembly of Learning Objects, in [30].

[16] Gaudioso E., Boticario J., User modeling on adaptive web-based learning communities, in Proceedings of the Knowledge-Based Intelligent Information and Engineering Systems, 7th International Conference, KES 2003, volume 2774 of Lecture Notes in Computer Science, Oxford UK, Springer-Verlag, 2003.

[17] Gilbert J., Han C., Adapting instruction in search of "a significant difference", Journal of Network and Computer Applications, 22:149-160, 1999.

[18] Gottwald S., Fuzzy Sets and Fuzzy Logic, Vieweg, 1993.

[19] Hatala M., Richards G., et al., The Interoperability of Learning Object Repositories and Services: Standards, Implementations and Lessons Learned, in [30].

[20] IEEE, Draft standard for learning object metadata, 2002.

[21] Lassila O., Swick R.R., Resource Description Framework (RDF) Model and Syntax Specification, W3C Recommendation, 1999.

[22] Martínez J.M., MPEG-7 Overview, ISO/IEC JTC1/SC29/WG11N5525, 2003.

[23] Mor E., Minguillon J., Dynamic generation of adaptive Internet-based courses, in [30].

[24] Nejdl W., Wolf B., et al., EDUTELLA: a P2P networking infrastructure based on RDF, in Proceedings of the 11th International World Wide Web Conference, ACM Press, 2002.

[25] Novák V., Perfilieva I., Mockor J., Mathematical Principles of Fuzzy Logic, Kluwer, 1999.

[26] Smith M.K., Welty C., McGuinness D.L., OWL Web Ontology Language Guide, W3C Recommendation, 2004. 
[27] Seebach J.A., Steen L., Counterexamples in Topology, 2nd edition, Springer-Verlag, 1978.

[28] Stash N., Cristea A., De Bra P., Authoring Learning Styles in Adaptive Hypermedia: Problems and Solutions, in [30].

[29] Sugiyama K., Hatano H., Yoshikawa M., Adaptive web search based on user profile constructed without any effort from users, in [30].

[30] Proceedings of the Thirteenth International World Wide Web Conference, ACM Press, 2004. 